\title{
Accurate Models of Energy Harvesting for Smart Environments
}

\author{
Greg Jackson, Sokratis Kartakis and Julie A. McCann \\ Imperial College London, London, United Kingdom \\ \{gregj, s.kartakis13, jamm\}@imperial.ac.uk
}

\begin{abstract}
Over the last decade, the energy optimization of resource constrained sensor nodes constitutes a major research topic in smart environments. However, state of the art energy optimization algorithms make strong and unrealistic assumptions of energy models, both in simulations and during the operation of smart systems. For instance, simplistic energy models for energy harvesting leads to inaccurate representation and prediction of the true dynamics of energy. Consequently, systems for smart environments are unable to meet expected performance criteria. In this paper, we propose innovative models to overcome the drawbacks of simplistic energy representations in smart environments. We provide the insights of how to generate precise lightweight energy models. Using the physical properties of solar and flow energy harvesting as case studies, the trade-off between energy harvesting inference and real-time measurement of energy generation is explored. To evaluate our proposed energy models against the simplistic versions, we use real measured data from our environmental micro-climate monitoring deployment in an urban park and a $103 \%$ improvement is seen. Additionally, to define the trade-offs between inferred and measured energy generation, experiments are conducted utilizing solar and smart water testbeds.
\end{abstract}

\section{INTRODUCTION}

Within smart environments, Wireless Sensor Network (WSN) technologies are utilized to monitor physical and environmental conditions [1]. A current major barrier to the adoption of WSN in smart environments is the availability of energy. Traditionally, deployments have been limited to locations where mains power is available or to a limited lifetime if using primary batteries. Recently, Energy Harvesting Wireless Sensor Networks (EH-WSN) obtain energy from sources such as solar, wind, thermal, vibration, and RF, coupled with resource management schemes, to overcome this issue [2].

With the introduction of energy harvesting, resource optimization methodologies moved from techniques which minimized energy consumed to techniques which seek to find a balance between the consumed and harvested energy. These energy aware algorithms are tested utilizing a combination of simulation, emulation and real world deployment. Yet, due to the costly and time consuming nature of real world experimentation, testing of energy aware algorithms in smart environments relies heavily on modeling and simulation [3] of energy. In parallel, monitoring of energy states in EH-WSN requires costly energy measurement circuitry or the ability to

This work is funded by the Intel Collaborative Research Institute (ICRI) for sustainable future cities. infer the energy state of the system. To perform these tasks, costly models of how energy harvesting and consumption impacts the energy storage state are needed.

Current research in EH-WSN seeks to effectively utilize energy using methods such as duty cycle adaptation [4], [5], duty cycle maximization [6], [7] and Energy Neural Operation (ENO) [8], [9]. Simulations of energy harvesting in this field tend to utilize simplistic solar energy models [10] to undertake simulations.

For the physical model proposed in this paper, the state of the art of energy generation [11], [12] is considered in order to generate a lightweight energy model. The inputs for this model can be provided by data from real world sources to represent energy generation dynamics accurately, such as the National Solar Radiation Database (NSRDB) [13].

This paper seeks to incorporate a representative understanding of the physical attributes of energy in smart environments. To begin, models of solar and wind energy are proposed to give a precise estimation of energy harvesting in smart environment simulations. Furthermore, the trade-offs of measurement and inference techniques for current generation during operation of an EH-WSN are explored.

The contributions of this paper can be summarized as follows:

- An innovative model is derived from the physical properties of solar and wind energy harvesting for use in smart environment simulations

- The trade-off analysis between inference and measurement of an energy harvester is explored utilizing solar and smart water network testbeds

The proposed models are evaluated by comparing data from real world experiments to standard simplistic models of energy harvesting. For each approach, estimations of reliability and cumulative errors are calculated in order to give metrics of performance.

The remaining sections of this paper are laid out as follows. Section II covers the proposed system model to derive energy generation from physical parameters. Section III presents the evaluation of this work for multiple EH-WSN scenarios and we conclude this paper in section IV.

\section{SySTEM MODEL}

In this paper, we consider the physical parameters of solar and wind energy harvesting in order to create an innovative 
lightweight model of energy generation for smart environments.

\section{A. Solar Energy Harvesting}

Solar energy harvesting is a well known source of energy for EH-WSN in smart environments. To create a physical model of solar energy harvesting, the power produced, $P_{p v}$ (in watts), of a solar cell is considered utilizing the work in [12].

$$
P_{p v}=\eta_{p v} A_{p v g} G_{t}
$$

where $\eta$ is the solar conversion efficiency, $A_{p v g}$ is the solar panel area and $G_{t}$ is the solar irradiation hitting a tilted solar plane, in $W / m^{2}$. Within solar energy generation models $\eta_{p v}$ is considered to be static. However, this is impacted by environmental conditions. To model changing environmental conditions impact on $\eta_{p v}$, the following is considered

$$
\eta_{p v}=\eta_{r} \eta_{p c}\left(1-\beta\left(T_{c}-T_{c r e f}\right)\right)
$$

where $\eta_{p c}$ is the solar cell efficiency efficiency, $\beta$ is the temperature coefficient, $\eta_{r}$ is the reference module efficiency, $T_{c}$ is the solar cell temperature and $T_{\text {cref }}$ is the reference cell temperature, both in ${ }^{\circ} \mathrm{C}$. To highlight the impact of this change, $\eta_{p v}$ is calculated over a 14 year period in Brasilia, Brazil.

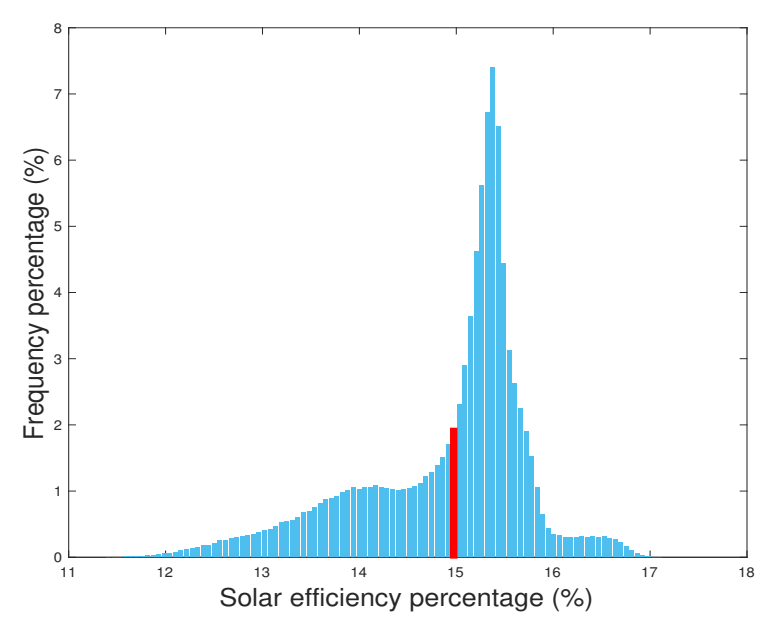

Figure 1: Changes in efficiency of solar cell, $\eta_{p v}$, due to environmental changes for an arbitrary environment in Brasilia, Brazil. The highlighted bar is the static efficiency.

In order to derive a more representative view of solar energy impinging on a surface, the conversion from incident solar energy to directed energy on an area can be calculated from physical parameters. Therefore, the work in [11] is considered, $G_{t}$ is the global irradiance on a tilted plane as is defined as

$$
G_{t}=G_{b n} \cos \theta+G_{d} R_{d}+\rho G R_{r}
$$

where the different elements of incoming solar energy can be expressed as $G_{b n}$, the direct normal irradiance, $E_{d}$, the diffuse horizontal irradiance and $G$, the global horizontal irradiance.
Furthermore, $\theta$ is the angle of incidence of the suns rays on the tilted plane, $R_{d}$ is the diffuse transposition factor, $\rho$ is the foreground albedo (or proportion of the incident light or radiation that is reflected by a surface), and $R_{r}$ is the transposition factor for ground reflection.

The output of this system model is a Physical Solar Energy Model (PSEM) which gives the ability to transform lighting data, available via open solar radiation databases for locations worldwide, to representative energy generation data for smart environment applications.

\section{B. Wind Energy Harvesting}

Another potential energy harvesting source for EH-WSN in the smart environments is wind power generation. Modeling of wind generation is non-trivial due to the non-linear response of wind turbines and quasi deterministic nature of wind production. From [12], [14], a model of wind energy production is derived where $P_{w}$ is the power contained in wind impinging on the surface of a micro wind turbine and is described as

$$
P_{w}=\frac{1}{2} \rho A V^{3}
$$

where $\rho$ is the air density at $20^{\circ} \mathrm{C}, A$ is the swept area of the rotor of the wind turbine and $V$ is the wind speed. From this usable power from the turbine, $P$, can be calculated.

$$
P=P_{w} c_{p}
$$

where $\mathrm{d} c_{p}$ is the maximum power coefficient of the wind turbine. Finally, there are conditions that need to be considered [12] e.g. the non linear nature of the wind turbine.

$$
P=\left\{\begin{array}{l}
P_{w} c_{p}, \text { if } V>V_{e} \\
0, \quad \text { otherwise }
\end{array}\right.
$$

where $V_{e}$ defines a minimum wind velocity over which the stall torque ${ }^{1}$ is overcome and the wind turbine begins producing energy, equally

$$
P=\left\{\begin{array}{l}
P_{w} c_{p}, \quad \text { if } V<V_{a} \\
0, \quad \text { otherwise }
\end{array}\right.
$$

where $V_{a}$ defines a maximum wind velocity over which producing energy could physically damage the wind turbine and is therefore turned off completely for safety.

Figure 2 gives an example of a wind energy profile derived utilizing the lightweight model presented here. This can be used as a representative input to wind EH-WSN simulations as, due to the immature nature of micro wind turbine technologies for smart environment applications, no standard method of simulating wind energy harvesting exists. Potentially, due to the lightweight nature of the proposed method, this can be used as a inferred energy generation sensor if real time wind data is available.

For an EH-WSN node, the introduction of current measurement circuitry to measure the energy harvested increases the

\footnotetext{
${ }^{1}$ The stall torque of a wind turbine is the torque produced when the output rotational speed is zero
} 


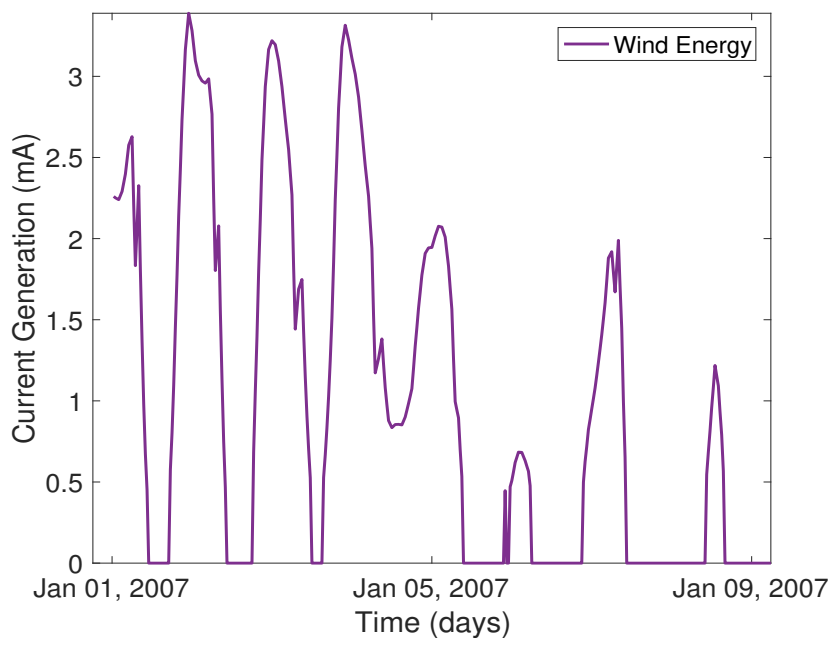

Figure 2: Example output of wind energy harvesting utilizing real wind speed data and model shown here.

energy consumption costs. This fact makes inferring energy generation an attractive solution. However, the accuracy of this approach varies depending on the use case scenario and adds complexity to the system in terms of computation. These trade-offs will be explored in the evaluation section.

\section{EvAluATION}

In this section, we present the results of experimental results for two scenarios of modeling and inference of energy harvesting in smart environments. To evaluate the efficacy of these scenarios, the experiments are conducted using real testbeds.

\section{A. Evaluation metrics}

As the methods of PSEM and inferred sensing estimate real data utilizing second order regression models, formal metrics are needed to validate their efficacy. To compare actual with estimated data from our models, we select as main metrics of reliability and error the following:

Estimation of reliability $\mathbf{R}^{\mathbf{2}}$ : To define formally the reliability of model fitting, $R^{2} \in[0,1]$, is used. This indicates the proportion of the variance between actual and estimated data (higher value represents a better model fit) and is calculated as follows:

$$
R^{2}=1-\frac{\sum_{i=1}^{N}\left(y_{i}-\hat{y}_{i}\right)^{2}}{\sum_{i=1}^{N}\left(y_{i}-\bar{y}\right)^{2}}
$$

where $y_{i}$ represents the real value, $\hat{y}_{i}$ the estimated value and $\bar{y}$ the mean of the $N$ measurements.

Normalized cumulative error $\mathbf{E}_{\mathbf{c}}(\mathbf{k})$ : The normalized cumulative error per interval $k, E_{c}(k)$, is utilized here to indicate the deviation between the actual and the estimated data. $E_{c}(k)$ is calculated as follows:

$$
E_{c}(k)=\frac{1}{Y_{\text {total }}} \sum_{i=1}^{k}\left|y_{i}-\hat{y}_{i}\right|, \quad \forall k \in[1, N]
$$

where $Y_{\text {total }}=\sum_{i}^{N} y_{i}$ represents the sum of all values, $y_{i}$ and $\hat{y}_{i}$ are the actual and estimated value of interval $i$ respectively, and $N$ defines the number of samples.

\section{B. Evaluation testbeds}

Three testbeds are used in this paper to evaluate our approaches:

Testbed 1 - Micro-Climate ENO (MCENO): This testbed consists of a twenty node micro-climate EH-WSN, Figure 3a, deployed in the wetlands of an urban park in London, UK, Figure $3 \mathrm{~b}$. The purpose of the testbed is to capture temperature, humidity, light and atmospheric pressure data in order to track changes in this ecologically diverse environment. From a EHWSN perspective, duty cycle maximization testing is being undertaken to understand the challenges to creating a WSN which can sustain itself through Energy Neutral Operation (ENO). This testbed is utilized in the modeling scenario.

Testbed 2 - Discrete Point Solar Testbed (DPST): Solar data is collected utilizing a DC power analyzer connected to a AM-1815 solar cell. Light levels are captured utilizing a TSL2561 light sensor. Testing is undertaken with variable intensity light source in order to capture the relationship between light levels and energy generation for an indoor EH-WSN application. This testbed provides solar energy harvesting data for the inference experiment.

Testbed 3 - WaterBox [15]: The WaterBox platform (see Figure $3 \mathrm{c}$ ) is developed to demonstrate real time monitoring and control by adapting innovative communication theories. WaterBox consists of a reservoir and three water supply areas. In the inlet of each supply area, a sensor/actuator (see Figure 3d) node is responsible to retrieve pressure and flow ${ }^{2}$ sensing data and control a valve. Further, a flow-based energy harvester is installed before each valve to harvest energy (see Figure 3d). To capture the total energy harvesting of each sensor/actuator node in real time, a current sensor is deployed. Similarly to testbed 2, WaterBox is used in the inference scenario.

\section{Solar Energy Simulation}

To validate the proposed system model, experimentation is undertaken using solar energy harvesting as an example use case. Figure 4 shows the comparison between:

- Simplistic Solar Energy Model (SSEM)

- PSEM approach proposed in this paper

- Ground Truth Solar Energy (GTSE) data

The SSEM utilizes an approximated solar energy source, the PSEM method utilizes our proposed model to derive energy generation and the real data, GTSE, is derived from the MCENO testbed. Using these methods solar energy generation data is calculated in order to understand the benefits and drawbacks of each approach. Figure 4 shows a comparison of the three approaches.

From Figure 4, it can be seen that, unlike the SSEM method, the PSEM and GTSE data take into account weather and

\footnotetext{
${ }^{2}$ The flow sensor is pulse based i.e. generate a pulse per $2.25 \mathrm{ml}$.
} 


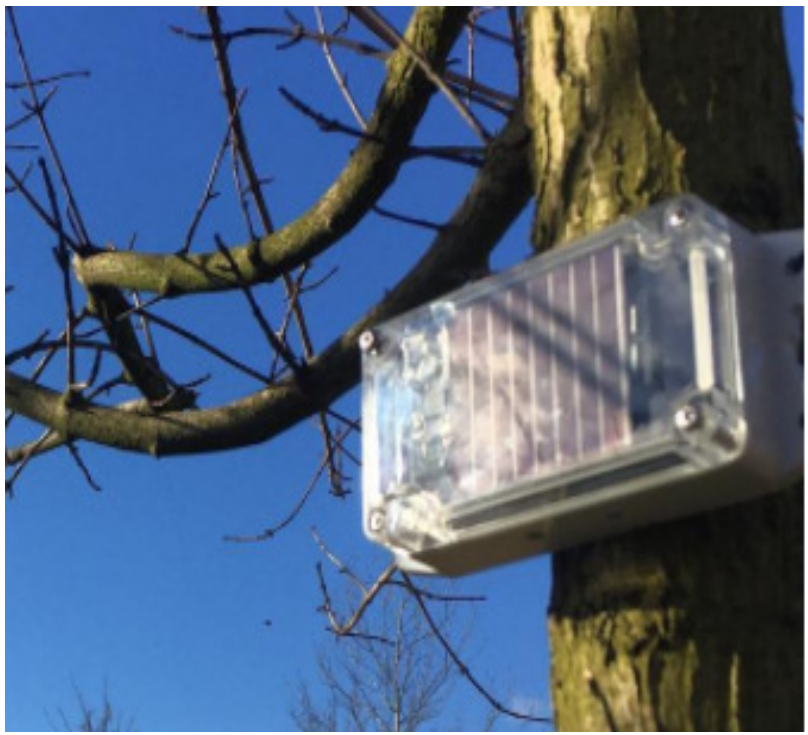

(a) Example Micro-climate sensor.

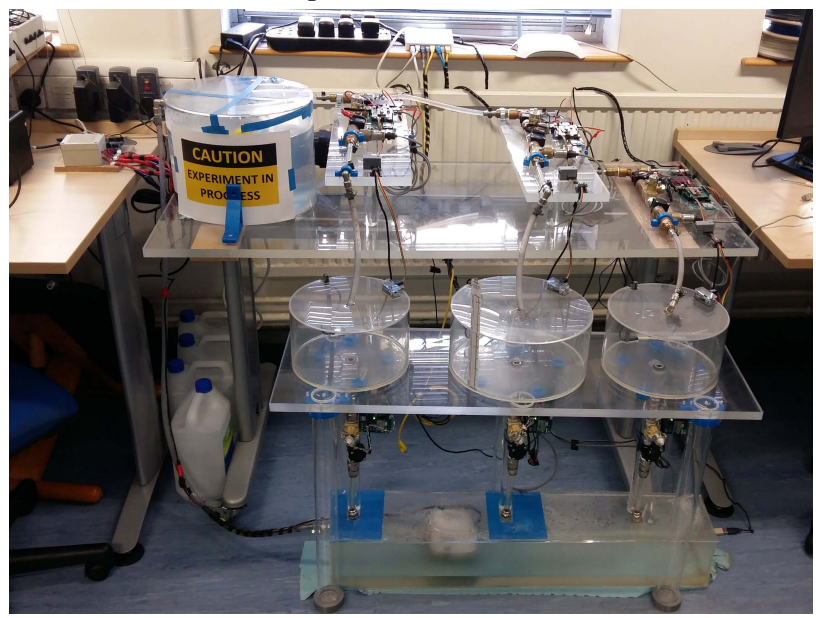

(c) WaterBox testbed structure.

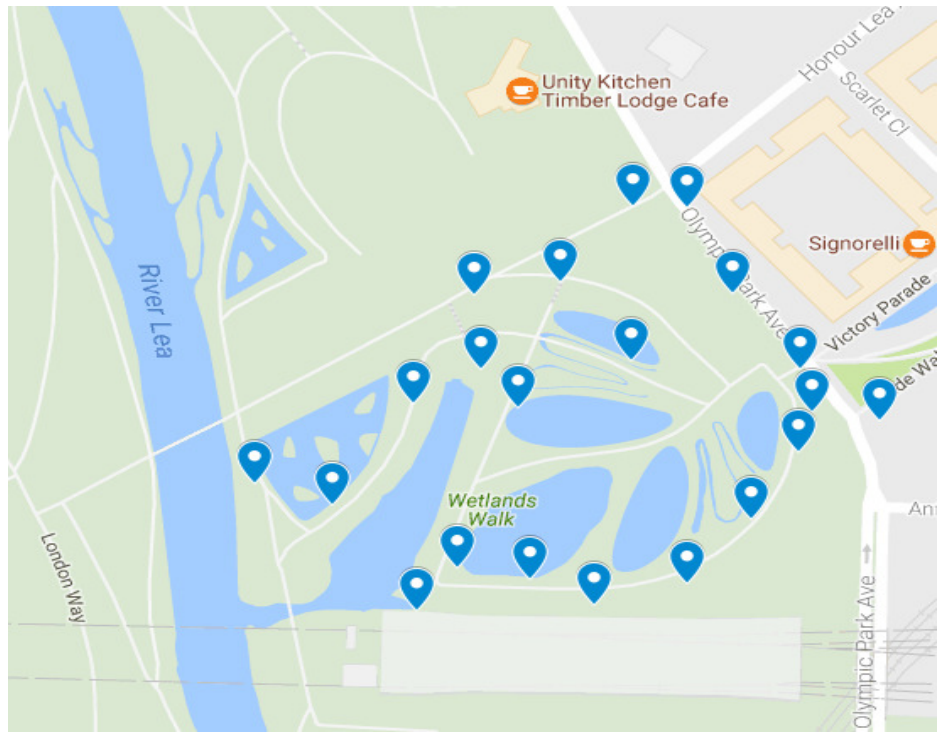

(b) Deployment locations.

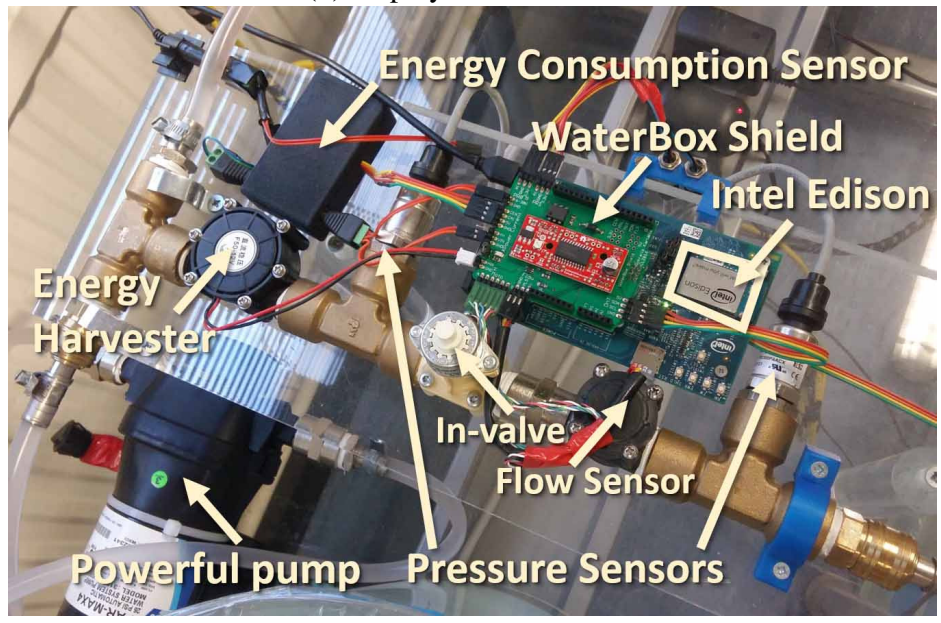

(d) Sensor and actuator node.

Figure 3: Micro-climate and smart water testbeds utilized in this paper

Table I: Results from simulation based testing.

\begin{tabular}{|c|c|c|}
\hline Method & $R^{2}$ & Total Current (mAh) \\
\hline \hline SSEM & 0.32 & 414.4 \\
\hline PSEM & 0.65 & 99.03 \\
\hline GTSE & 1 & 121.03 \\
\hline
\end{tabular}

seasonal changes. From Table. I, the actual $R^{2}$ value and total current generated reveals that the PSEM model outperforms the SSEM model. Specifically, the PSEM method has a $103 \%$ improvement over the SSEM in terms of estimation of reliability. The current generated by each approach is also calculated to understand the overall variation from the real world measurements. As the SSEM does not take into account seasonal and environmental changes it overestimates the total energy generated by $241 \%$, whereas the PSEM underestimates the total energy generation by $18 \%$. Figure 5 highlights the normalized cumulative error of the SSEM and PSEM method when compared to the GTSE method. The error of the PSEM approach is $15 \%$ of the error of the SSEM method, highlighting the benefit of the PSEM method.

There are trade-offs to recording real solar data (GTSE) and utilizing the PSEM model. The PSEM does not precisely match an energy generation profile (GTSE) of a sensor node in a smart environment due to the influence of local factors such as local shading. Conversely, as the PSEM method is generated from publicly available data sources with years of historical data [13], it can create energy profiles for nodes in multiple different environments in order to easily test the robustness of an algorithm; data that would be very time intensive to gather manually. Therefore, depending on the needs of an evaluation the appropriate methodology can be chosen. Considering real world deployments, EH-WSN requires a precise measurement of the energy generated measurement. The ability to infer these measurements is discussed in the following section. 


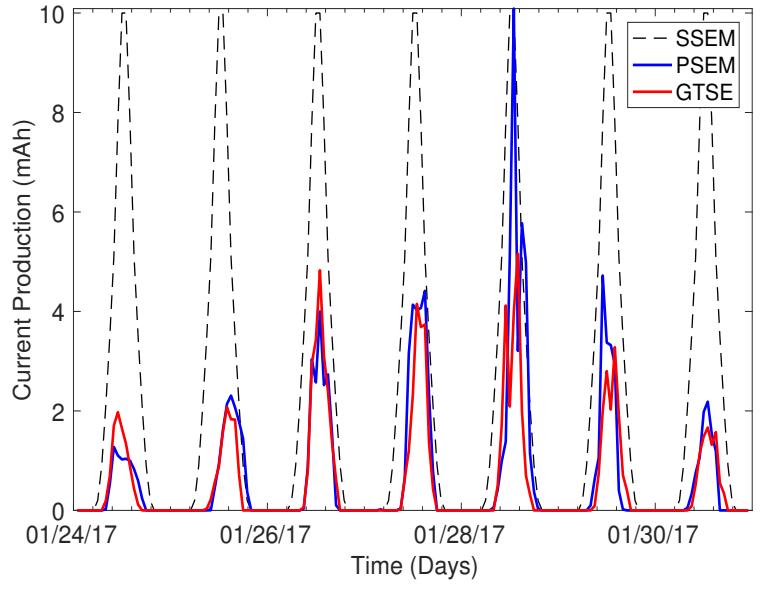

Figure 4: Comparison of SSEM, PSEM and GTSE solar energy harvesting data for a one week test in an urban park, London, UK.

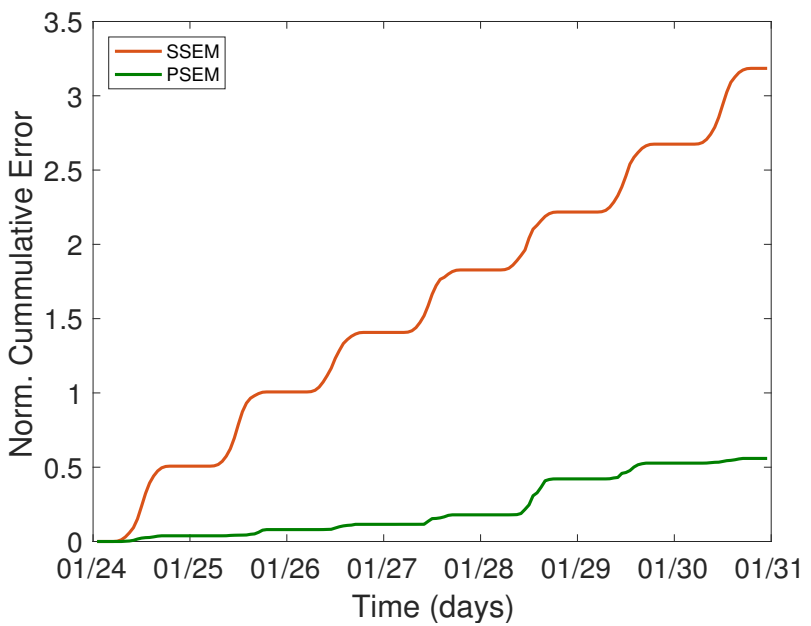

Figure 5: Normalized cumulative error of estimation for PSEM and SSEM method compared to GTSE data

\section{Inference based sensing}

To explore the ability to infer energy generation based on environmental sensing data, two different sets of experiments are conducted by exploiting real testbed infrastructure. The first set examines the estimation of solar energy harvesting generated by using the DPST testbed. A second experiment, utilizing the WaterBox testbed, is proposed to test the ability to infer energy generation from other energy harvesting sources, in this case inferring flow based energy harvesting from a flow sensor.

To infer solar energy harvesting, a second order regression model is exploited to derive the relationship between a TSL2561 light sensor and solar energy harvesting; the results can be seen in Figure 6. Based on this experiment, the estimation of reliability is calculated to be $R^{2}=0.99$ which indicates that energy harvesting data based on solar can be inferred accurately utilizing a light sensor.

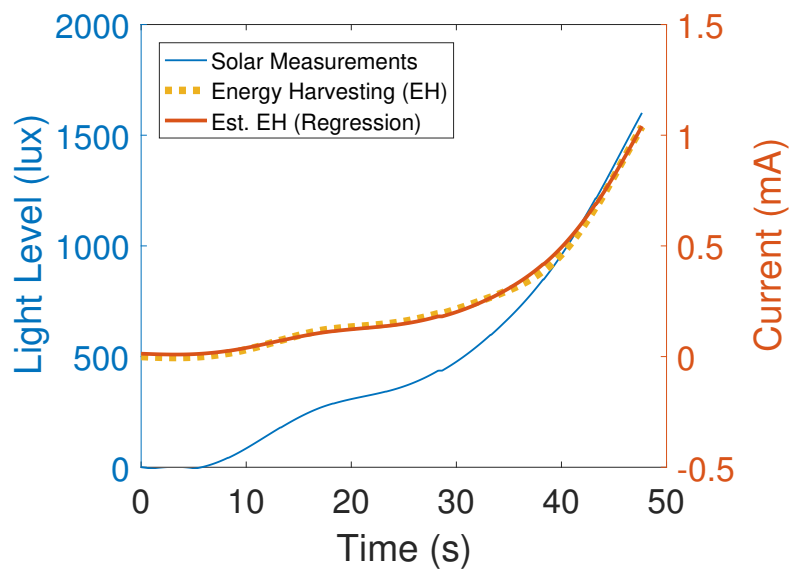

Figure 6: Inferring solar energy harvesting based on a TSL2561 light sensor.

In the second set of experiments the inference method is tested for a another smart environment; namely a smart water network. Using the WaterBox testbed, the inference method is applied to a flow based energy harvester to explore the ability of flow sensors to infer current generation in smart water applications.

Figure $7 \mathrm{a}$ illustrates real flow rate and energy harvesting measurements from a WaterBox node with inter-sampling period $5 \mathrm{msec}$. In contrast to solar data of the previous experiment, flow rate is a noisy signal making the inference of energy harvesting problematic. To reduce the noise without sacrificing accuracy, we apply filters which reduce the sample rate from 200 samples of flow rate per second to 1.45 samples per second; this output can be seen in Figure $7 \mathrm{~b}$. Once the data has been filtered, a regression model is trained to find the relationship between flow rate and energy harvesting data, the accuracy of the resulting model was dramatically low i.e. $R^{2}=0.08$. To improve the estimation reliability, a moving average smoothing technique is applied to low frequency flow rates before the generation of the regression model. Despite this extra step, the reliability of the new regression model only increases to $R^{2}=0.14$, the result of both approaches can be seen in Figure $7 \mathrm{~b}$.

The result of the two sets of experiments reveal that the accuracy of energy harvesting inference varies depending the application and available sensors. To provide quantitative comparison between the two scenarios, i.e. solar and water flow, Figure 8 illustrates the normalized cumulative error. Based on Figure 8, the cumulative error of energy harvesting inference for solar application is one order of magnitude less than the water flow. Specifically, based on the quantitative results of Table II, the inference based on solar results in $17 \%$ of the error of the flow based inference method.

Based on the above analysis, the energy harvesting estima- 


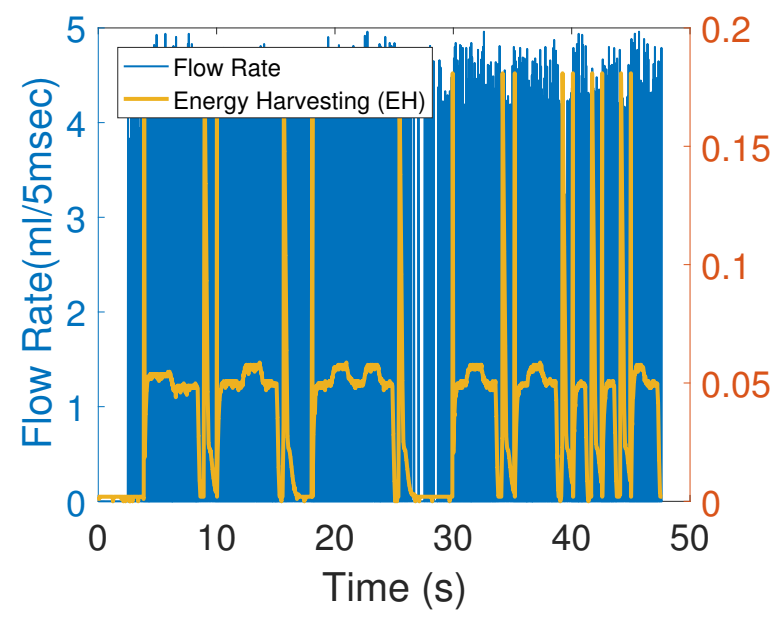

(a) Flow rate and energy harvesting raw data $(5 \mathrm{msec})$.

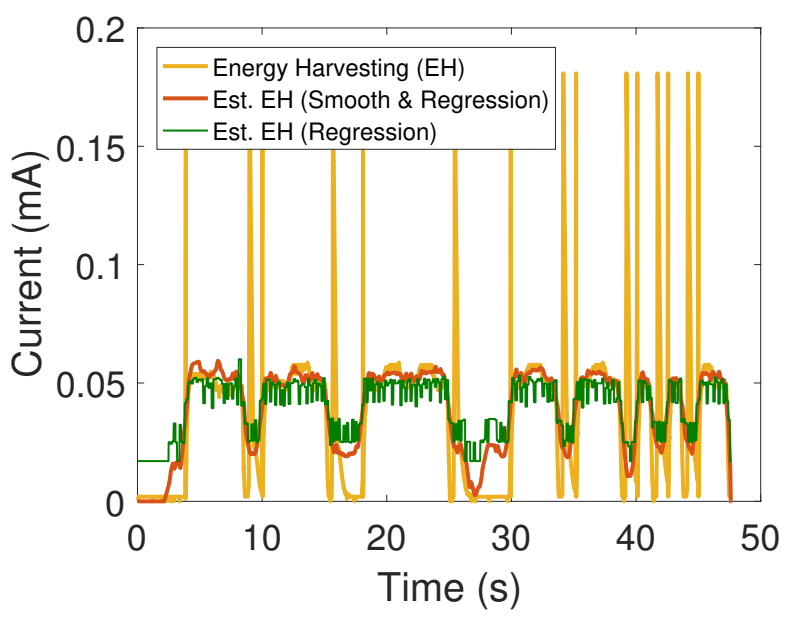

(b) Energy harvesting estimation results.

Figure 7: Inferring energy harvesting based on water flow.

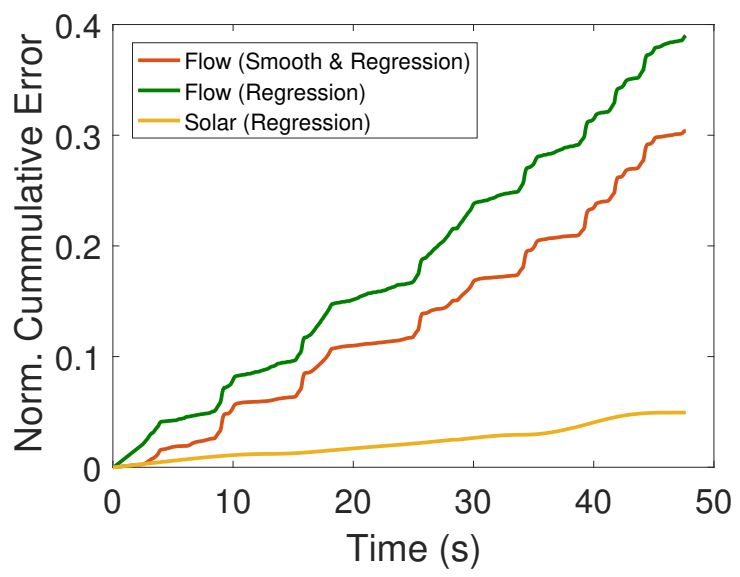

Figure 8: Normalized cumulative error of estimation for inference method.

Table II: Quantitative evaluation results.

\begin{tabular}{|c|c|c|c|c|}
\hline $\begin{array}{c}\text { Energy } \\
\text { Harvesting }\end{array}$ & $\begin{array}{c}\text { Inference } \\
\text { Technique }\end{array}$ & $\begin{array}{c}\text { Inference } \\
\text { Reliability } \\
\left(\mathbf{R}^{\mathbf{2}}\right)\end{array}$ & $\begin{array}{c}\text { Total } \\
\text { Cumul. Error } \\
(\text { Absolute mA) }\end{array}$ & $\begin{array}{c}\text { Total } \\
\text { Cumul. Error } \\
\text { (Normalized) }\end{array}$ \\
\hline \hline Solar & Regression & 0.99 & 1178 & 0.05 \\
\hline Water Flow & Regression & 0.08 & 1465 & 0.39 \\
\hline Water Flow & Smoot \& Regres. & 0.14 & 1142 & 0.30 \\
\hline
\end{tabular}

tion performance depends on the application and the behavior of the harvesting element. For example, in the case of solar, the estimation reliability is high and the solar energy sensor can be avoided by using only data from the light sensor to infer both light intensity and energy generation. On the other hand, in the case of water flow, the inference techniques is unable to reliably estimate energy production from water flow sensing. As a result, the existence of both water flow and energy harvesting current sensors is unavoidable.

\section{CONCLUSION}

Smart environments aim to achieve the goal of perpetually powered EH-WSN powered through intelligent management of energy scavenged from the environment. However, the lack of accurate models of energy generation in smart environments results in energy being under or overestimated, both in simulation and during operation of EH-WSN. In this paper, we not only propose a better model by considering the physical properties of energy harvesting devices but also explore the trade-offs of inferring and measuring energy generation. By conducting experiments on real testbeds, evaluation results reveal that our proposed innovative lightweight model outperforms traditional methods in terms of reliability by $103 \%$.

To answer the question "can energy generation be accurately inferred in EH-WSN?" experiments undertaken on two testbeds conclude that the accuracy of this method is dependent on application scenario. For solar energy inference utilizing light sensing data a reliability of $R^{2}=0.99$ is found highlighting the benefits of this approach. On the other hand, inference based on flow energy harvesting was determined to be unsuitable with a reliability of $R^{2}=0.14$.

\section{REFERENCES}

[1] P. Friess, Internet of things: converging technologies for smart environments and integrated ecosystems. River Publishers, 2013.

[2] S. Sudevalayam and P. Kulkarni, "Energy harvesting sensor nodes: Survey and implications," IEEE Communications Surveys and Tutorials, vol. 13, no. 3, pp. 443-461, 2011.

[3] T. W. Hnat, V. Srinivasan, J. Lu, T. I. Sookoor, R. Dawson, J. Stankovic, and K. Whitehouse, "The hitchhiker's guide to successful residential sensing deployments," Proceedings of the 9th ACM Conference on Embedded Networked Sensor Systems - SenSys '11, p. 232, 2011. [Online]. Available: http://dl.acm.org/citation.cfm?doid=2070942. 2070966

[4] T. N. Le, A. Pegatoquet, O. Sentieys, O. Berder, and C. Belleudy, "Dutycycle power manager for thermal-powered wireless sensor networks," IEEE International Symposium on Personal, Indoor and Mobile Radio Communications, PIMRC, pp. 1645-1649, 2013. 
[5] C. M. Vigorito, D. Ganesan, and A. G. Barto, "Adaptive control of duty cycling in energy-harvesting wireless sensor networks," 2007 4th Annual IEEE Communications Society Conference on Sensor, Mesh and Ad Hoc Communications and Networks, SECON, pp. 21-30, 2007.

[6] Z. Liu, X. Yang, S. Yang, and J. a. McCann, "Efficiency-Aware: Maximizing Energy Utilization for Sensor Nodes Using PhotovoltaicSupercapacitor Energy Systems," pp. 1-11, 2013. [Online]. Available: http://www.hindawi.com/journals/ijdsn/2013/627963/

[7] D. K. Noh and K. Kang, "Balanced energy allocation scheme for a solarpowered sensor system and its effects on network-wide performance," Journal of Computer and System Sciences, vol. 77, no. 5, pp. 917-932, 2011. [Online]. Available: http://dx.doi.org/10.1016/j.jcss.2010.08.008

[8] A. Kansal, J. Hsu, S. Zahedi, and M. B. Srivastava, "Power Management in Energy Harvesting Sensor Networks," vol. 6, no. 4, pp. 1-38, 2007.

[9] S. Peng and C. P. Low, "Prediction free energy neutral power management for energy harvesting wireless sensor nodes," Ad Hoc Networks, vol. 13, no. PART B, pp. 351-367, 2014. [Online]. Available: http://dx.doi.org/10.1016/j.adhoc.2013.08.015

[10] E. Lattanzi, E. Regini, A. Acquaviva, and A. Bogliolo, "Energetic sustainability of routing algorithms for energy-harvesting wireless sensor networks," Computer Communications, vol. 30, no. 14-15, pp. 2976-
2986, 2007.

[11] S. Wilcox, "National Solar Radiation Database 1991-2005 Update: User's Manual," Instrumentation, no. April, p. 472, 2007. [Online]. Available: http://www.osti.gov/energycitations/product.biblio. jsp?osti $\left\{\backslash \_\right\}$id $=901864$

[12] B. Bhandari, S. R. Poudel, K.-T. Lee, and S.-H. Ahn, "Mathematical modeling of hybrid renewable energy system: A review on small hydro-solar-wind power generation," International Journal of Precision Engineering and Manufacturing-Green Technology, vol. 1, no. 2, pp. 157-173, 2014. [Online]. Available: http://link.springer.com/10.1007/ s40684-014-0021-4

[13] NREL, "NSRDB- National Solar Radiation Data Base." [Online]. Available: http://rredc.nrel.gov/solar/old $\left\{\backslash_{-}\right\}$data/nsrdb/

[14] E. Bolte and M. Landwehr, "Mathematical model of small wind turbines," 2014 Ninth International Conference on Ecological Vehicles and Renewable Energies (EVER), no. 2, pp. 1-6, 2014. [Online]. Available: http://ieeexplore.ieee.org/lpdocs/epic03/wrapper. htm?arnumber $=6844151$

[15] S. Kartakis, E. Abraham, and J. A. McCann, "Waterbox: A testbed for monitoring and controlling smart water networks," in Proc. ACM CysWater, CPSWeek, 2015, p. 8. 